\title{
A mutant of Neurospora crassa that has a long lag phase in low-calcium medium
}

\author{
Takuji OHnishi, Gerd CoRnelius $\dagger$ and Hideaki NaKashima* \\ Department of Biology, Faculty of Science, Okayama University, Okayama, Japan 700
}

(Received 2 January 1992; revised 15 April 1992; accepted 5 May 1992)

\begin{abstract}
A mutant of Neurospora crassa that has a long lag phase when grown in medium that contains less than $10 \mu \mathrm{M}$ $\mathrm{CaCl}_{2}$ was isolated from UV-irradiated conidia. However, the rate of uptake of calcium ions by mycelium and the dependence on magnesium ions of the growth of this mutant were the same as those of the wild-type strain. The phase of the circadian clock, within the entire circadian cycle of the mutant, could not be shifted by exposure to fluorescent white light during growth in liquid medium. However, a brief incubation at high temperature resulted in a large shift in phase, and the phase response curve for the mutant was similar to that for the wild-type strain. The mutant strain had the same sensitivity to light as the wild-type strain with respect to carotenoid synthesis. The relevant mutation may affect the process involved in transduction of signals from the light-perception system to the clock mechanism and this process may be related to calcium homeostasis in Neurospora cells.
\end{abstract}

\section{Introduction}

Circadian rhythms, endogenous diurnal fluctuations in biological activities, are well known in various organisms from lower eukaryotic plants to human beings. However, the molecular mechanism responsible for circadian rhythms, the circadian clock, is not well understood. In Neurospora, we found that the calcium ionophore A23187 and two calmodulin antagonists, W5 and W12, shifted the phase of the circadian conidiation rhythm and that phase shifting by A23187 was inhibited by calcium ions. We concluded that the metabolism of calcium is important for clock functions from the late subjective day phase to the early subjective night phase (Nakashima, 1984, 1986). Important roles for calcium in clock functions have also been indicated in other organisms, such as Aplysia (Eskin \& Corrent, 1977) and Euglena (Goto et al., 1985; Tamponnet \& Edmunds, 1991).

The relationship of calcium ions to the circadian clock remains urıknown. To further our understanding of the relationship between clock function and the metabolism of calcium, we attempted to isolate mutants with phenotypic differences in the metabolism of calcium, assuming that growth of these mutants would vary with different concentrations of calcium ions in the culture

\footnotetext{
* Author for correspondence. Tel. 0862521111 ; fax 0862526601 .

$\dagger$ Present address: Institute for Genetics, University of Saarbrucken, D-6600 Saarbrucken, FRG.
}

medium. If clock function and the metabolism of calcium are closely correlated, these mutants should show different characteristics with respect to various parameters of clock function, such as period length, sensitivity to light and temperature compensation. We succeeded in isolating two different types of mutants: one type that cannot grow in medium that contains a normal concentration of calcium ions (close to $1 \mathrm{~mm}$ ) and the other that cannot grow in medium that contains a low concentration of calcium ions (less than $10 \mu \mathrm{M}$ ). Two mutant strains of the former type have been described previously (Cornelius \& Nakashima, 1987). In this report, we describe a mutant strain that is of the latter type. This strain exhibits various physiological differences when compared to the wild-type strain. In addition, our results suggest that calcium ions may function in the process that connects the system involved in perception of light to the clock mechanism.

\section{Methods}

Strain and mutagenesis. The band $(b d)$ strain was used in all experiments as the wild-type strain. Stock cultures were maintained on complete medium (Horowitz, 1947) containing agar. Isolation of mutants was carried out by the method described by Davis \& de Serres (1970), with the slight modifications that have been reported previously (Cornelius \& Nakashima, 1987). Mutant strains that were isolated were backcrossed twice with the ca- $23-2^{+}$strain, and progeny that had the same phenotype as each isolated mutant were isolated and used in subsequent experiments. 
Uptake of ${ }^{45} \mathrm{Ca}^{2+}$ ions. Mycelia were grown in liquid medium containing Vogel's salts (Vogel, 1956) and $2 \%(\mathrm{w} / \mathrm{v})$ sucrose as described elsewhere (Slayman \& Slayman, 1979), and were suspended in 50 mM-MOPS buffer (pH 5.8) with $0.3 \%$ glucose. ${ }^{45} \mathrm{Ca}^{2+}$ as $\mathrm{CaCl}_{2}$ was added to give a final radioactivity of $1 \mu \mathrm{Ci} \mathrm{ml}^{-1}\left(37 \mathrm{kBq} \mathrm{ml}^{-1}\right)$ and, after given times, mycelia were harvested and washed twice with $5 \mathrm{ml}$ ice cold $5 \mathrm{~mm}-\mathrm{CaCl}_{2}$ on filter paper that had been prewetted with the same solution. After drying, mycelia were counted by liquid scintillation

Extraction and assay of carotenoid. Quantification of carotenoid in mycelia was performed by the method reported by Harding (1974), with a slight modification. Conidia $\left(4 \times 10^{5}\right.$ in the case of ca-23-2 $-2^{+}$, and $8 \times 10^{5}$ in the case of ca-23-2) were inoculated in disposable Petri dishes ( $35 \mathrm{~mm}$ diam.) with $4 \mathrm{ml}$ medium containing Vogel's salts, $2 \%$ sucrose and $0.8 \%$ Tween 80 . Conidia were then cultured at $18{ }^{\circ} \mathrm{C}$ for $6 \mathrm{~d}$ in continuous darkness. After the removal of excess culture medium into filter paper, three mycelial mats were transferred to a disposable Petri dish $(100 \mathrm{~mm}$ diam.) with $20 \mathrm{ml}$ of the same medium, supplemented with $0.5 \%$ sorbose, and incubated for $2 \mathrm{~h}$ at $26^{\circ} \mathrm{C}$. The mats were irradiated with 3000 lux of white light for different times and cultured for $2 \mathrm{~d}$ at $6{ }^{\circ} \mathrm{C}$ in continuous darkness. Extraction and measurements were carried out as reported by Harding (1974). After extraction of carotenoid, the residue was dried and treated with $5 \mathrm{ml}$ $0.5 \mathrm{M}-\mathrm{NaOH}$ for $30 \mathrm{~min}$ at $95^{\circ} \mathrm{C}$ for extraction of total protein. Protein was assayed by the method of Bradford (1976).

Determination of conidiation rhythm. The liquid culture method was similar to that reported previously (Nakashima, 1981). Conidia $(1.3 \times$ $10^{6}$ in the case of ca-23-2+, $10^{7}$ in the case of the mutant ca-23-2) were suspended in distilled water and inoculated into $25 \mathrm{ml}$ liquid medium containing Vogel's salts, $0.3 \%$ glucose and $0.5 \%$ arginine, and cultured in Petri dishes for about $36 \mathrm{~h}$ at $25^{\circ} \mathrm{C}$ in continuous light. The mycelial mats were cut with a cork-borer, $10 \mathrm{~mm}$ diameter, and 6 discs were transferred to $125 \mathrm{ml}$ Erlenmeyer flasks, each containing $25 \mathrm{ml}$ culture medium with Vogel's salts, $0.03 \%$ glucose and $0.05 \%$ arginine. Specimens were cultured in continuous darkness, treated with a brief exposure to light or high temperature at different times and transferred individually to race tubes containing $8 \mathrm{ml}$ culture medium. The medium contained Vogel's salts, $0.1 \%$ glucose, $0.17 \%$ arginine and $1.5 \%$ agar, in the case of the ca- $23-2^{+}$strain, and Vogel's salts, $2 \%$ glycerol and $1.5 \%$ agar in the case of ca-23-2. The phase, or the time at which the first conidial banding was evident in race tubes, was calculated by linear regression analysis (Dharmananda \& Feldman, 1979).

\section{Results}

\section{Growth and rate of uptake of calcium ions}

We succeeded in isolating ca-23-2 after screening about 4000 colonies that were unable to grow in culture medium containing A23187 and high concentrations $(5 \mathrm{mM})$ of calcium ions. When mycelial growth was examined $48 \mathrm{~h}$ after inoculation, ca-23-2 was found to have grown poorly in culture medium that contained less than $100 \mu \mathrm{M}-\mathrm{CaCl}_{2}$ (Fig. 1). The optimum concentration of calcium ions for growth of ca-23-2 was close to that for ca-23-2+ and was around $1 \mathrm{mM}$. Vogel's medium contains $0.7 \mathrm{mM}-\mathrm{CaCl}_{2}$. The growth of ca- $23-2+$ fell to only $60 \%$ of the maximum even at $10 \mu \mathrm{M}-\mathrm{CaCl}_{2}$. By contrast, the dependence of mycelial growth on the external concentration of magnesium ions was the same for both strains.

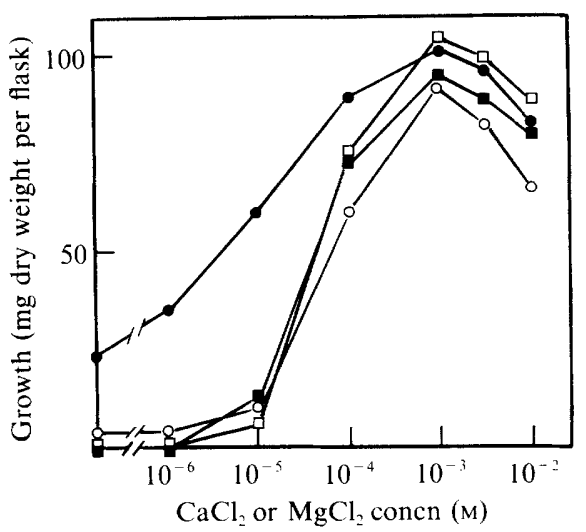

Fig. 1. Effects of various concentrations of calcium and magnesium ions on mycelial growth of Neurospora. Conidia $\left(2 \times 10^{6}\right)$ of ca-23-2+ (filled symbols) and ca-23-2 (open symbols) were inoculated into $20 \mathrm{ml}$ culture medium with different concentrations of $\mathrm{CaCl}_{2}$ (circles) or $\mathrm{MgCl}_{2}$ (squares) and cultured for $48 \mathrm{~h}$ with shaking at $26^{\circ} \mathrm{C}$. Since $\mathrm{MgSO}_{4}$ is included in Vogel's salts, sulphate ions were added as $\mathrm{Na}_{2} \mathrm{SO}_{4}$ at the same concentration as that in Vogel's salts.

The growth rate of ca-23-2 was a little lower than that of ca-23-2+, even when ca-23-2 was cultured in medium that contained the optimum concentration of calcium ions (1 mM; Fig. 2). However, ca-23-2 had a very long lag phase in culture medium that contained $10 \mu \mathrm{M}-\mathrm{CaCl}_{2}$. After the lag period ca-23-2 grew at a similar rate to that in medium containing $1 \mathrm{mM}-\mathrm{CaCl}_{2}$. Whenever $1 \mathrm{mM}$ $\mathrm{CaCl}_{2}$ was added to the culture medium during the lag phase, mycelium began to grow rapidly after several hours (Fig. 2).

Fig. 3 shows the distribution of progeny from a backcross of ca-23-2 to ca-23-2+ and illustrates the approximately $1: 1$ segregation among random spores. The growth of the ca-23-2 strain in the progeny of this backcross was always inhibited in medium with $10 \mu \mathrm{M}$ $\mathrm{CaCl}_{2}$ and this strain also gave no conidial banding on agar-solidified medium that contained glucose as the carbon source. Poor conidial bands were observed only when glucose was replaced by $2 \%$ glycerol or acetate.

The rate of uptake of calcium ions by the mycelium of ca-23-2 was the same as that of ca-23-2+ when both strains were cultured in a medium containing $1 \mathrm{mM}$ $\mathrm{CaCl}_{2}$ (Fig. 4). In both strains, the maximum rate of uptake was about $0.3 \mathrm{nmol} \mathrm{min}^{-1}(\mathrm{mg} \text { protein })^{-1}$.

\section{Carotenoid content and phase response curves}

Light-induced synthesis of carotenoid was measured in strains ca-23-2 and ca-23-2+ (Fig. 5). Carotenoid synthesis occurred maximally after $2 \mathrm{~min}$ of irradiation with white light in both strains. The two strains have almost the same sensitivity to light with respect to stimulation of the synthesis of carotenoid. However, the maximum 


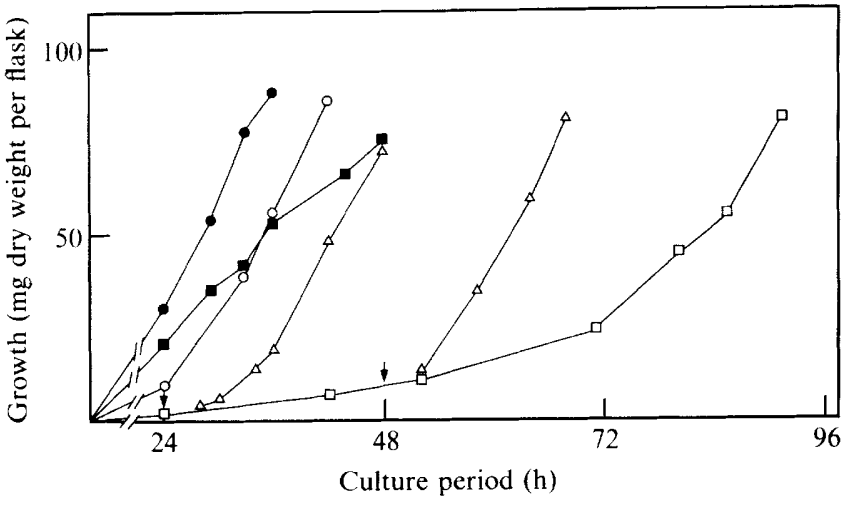

Fig. 2. Growth of strains ca-23-2+ and ca-23-2 at different concentrations of calcium ions. The procedure was the same as that described in the legend to Fig. 1. Mycelia were harvested at different times, as indicated. Two strains, ca-23-2+ (filled symbols) and ca-23-2 (open symbols), were cultured in medium that contained $1 \mathrm{~mm}$ $\mathrm{CaCl}_{2}$ (circles) or $10 \mu \mathrm{M}-\mathrm{CaCl}_{2}$ (squares). $\mathrm{CaCl}_{2}$ was added to a final concentration of $1 \mathrm{~mm}$ at different times as indicated by arrows (triangles).

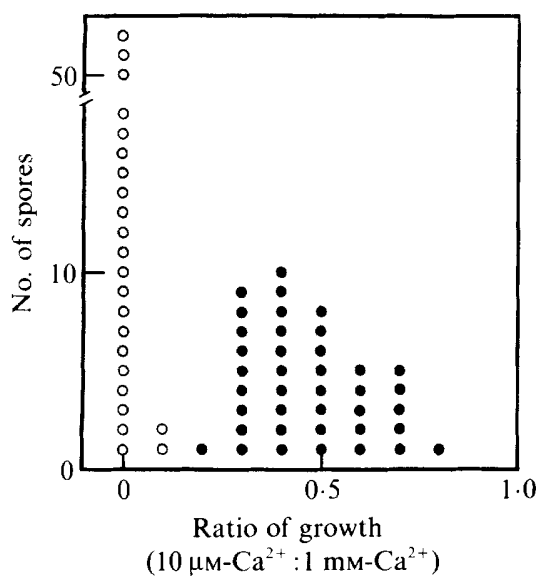

Fig. 3. Distribution of numbers of spores among progeny of a cross between ca-23-2 and ca-23-2+. The same amounts of conidia were inoculated into aliquots of medium that contained $10 \mu \mathrm{M}-$ or $1 \mathrm{mM}-$ $\mathrm{CaCl}_{2}$ and cultured for $1 \mathrm{~d}$. Samples of conidia were inoculated into race tubes containing medium with glucose as the carbon source and cultured for $7 \mathrm{~d}$ in continuous darkness. Conidiation; $O$, no conidiation.

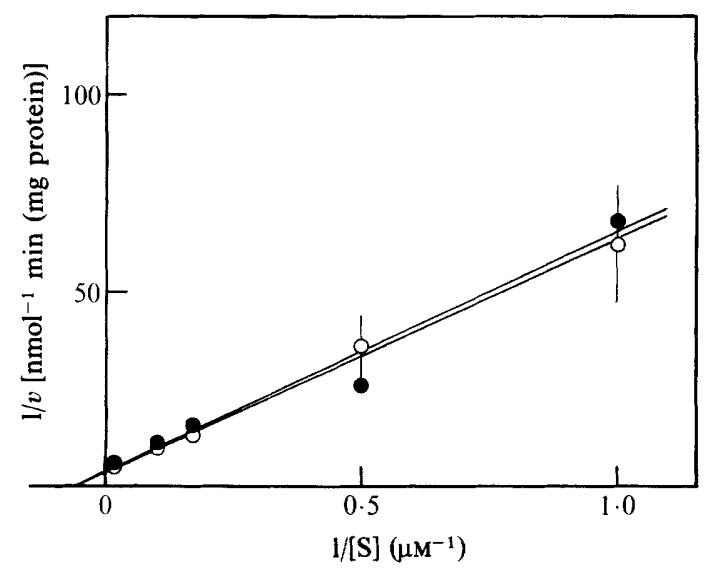

Fig. 4. Lineweaver-Burk plot of calcium uptake as a function of the concentration of extracellular calcium ions. Mycelia of both strains, ca$23-2^{+}(O)$ and ca-23-2 $(O)$, were incubated for $10 \mathrm{~min}$ in media containing different concentrations of $\mathrm{CaCl}_{2}$. level of carotenoid after saturating irradiation in the wild type was twice that in ca-23-2.

To examine phase shifting by light and high temperature, mycelia of both strains were cultured in liquid medium containing very low concentrations of glucose and arginine. In this medium, they failed to grow until they were transferred to agar-solidified medium containing a normal concentration of a carbon source, namely glucose or glycerol. Since ca-23-2 did not make conidial bands in the agar medium containing glucose, this strain was cultured in glycerol-containing agar medium. Preliminary experiments showed that the sensitivity of ca$23-2+$ to light in glucose-containing medium was exactly the same as that in glycerol-containing agar medium. Therefore, this strain was cultured in the former medium because conidial banding in glucose-containing medium was much clearer than in glycerol-containing medium. Phase shifting by light was examined from 24 to $72 \mathrm{~h}$ after a light-dark transition in both ca-23-2+ and ca-23-2 strains (Fig. 6). A cross-over point from maximum

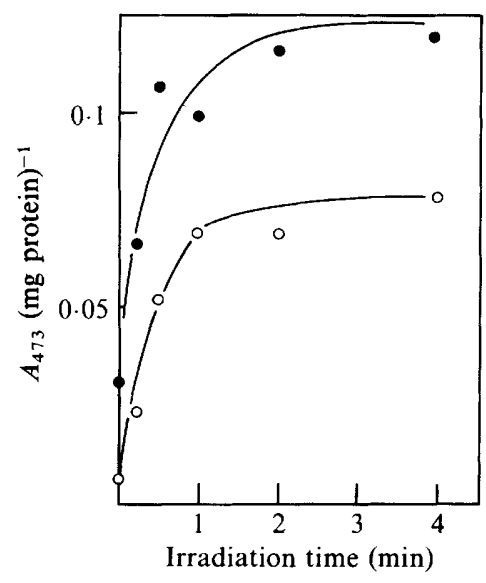

Fig. 5. Carotenoid content of mycelia after light irradiation. Mycelia of ca-23-2+ $(O)$ and ca-23-2 (O) were irradiated with fluorescent white light (3000 lux) for different times and assayed for carotenoid synthesis. 


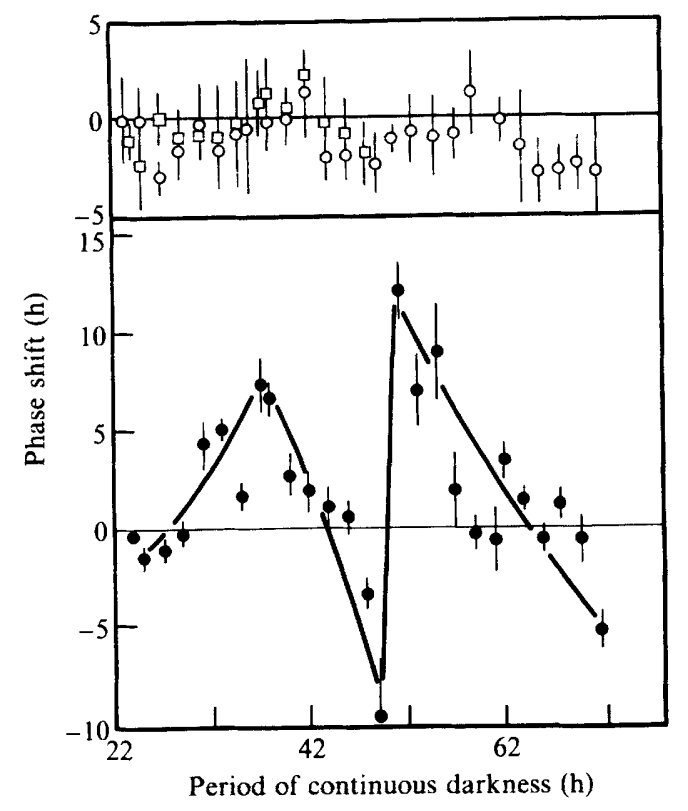

Fig. 6. Phase response curves for light. Mycelial discs of ca-23-2+ (filled symbols) and ca-23-2 (open symbols) were cultured in continuous darkness and irradiated with fluorescent white light ( 3000 lux) for $5 \mathrm{~min}$ (circles) or $15 \mathrm{~min}$ (squares) at different times between 24 and $72 \mathrm{~h}$ after inoculation. After 48 or $72 \mathrm{~h}$ all discs were transferred individually to race tubes. The phases were compared with those of controls which were not irradiated with light. Error bars indicate standard deviations of results from six different race tubes.

phase-delay to maximum phase-advance appeared $29 \mathrm{~h}$ and $50 \mathrm{~h}$ after the light-dark transition, the times being separated by about $21 \mathrm{~h}$ in the case of ca-23-2+. This value was almost equal to the period length of the conidiation rhythm. The same experiments were performed with the mutant ca-23-2, which did not show any regular shifting of phase by light from 24 to $72 \mathrm{~h}$. The experiment was repeated using a threefold longer period of irradiation with light, but ca-23-2 still did not show any regular shifting of phase by light. To examine the correlation between inhibition of growth in low-calcium $(10 \mu \mathrm{M})$ medium and insensitivity to light of ca-23-2, 18 progeny from the experiments for which results are shown in Fig. 3 were picked and their sensitivity to light was examined (Fig. 7). The progeny with the same phenotype as ca-23-2 did not show any regular phase shifting by light. However, progeny in which growth was not inhibited by low-calcium $(10 \mu \mathrm{M})$ medium exhibited a large shift in phase when they were irradiated with light from 48 to $54 \mathrm{~h}$ after the light-dark transition.

In contrast to the results with light, brief exposure to high temperature $\left(35^{\circ} \mathrm{C}\right)$ did shift the phase, dependent on the phase at which samples were treated (Fig. 8), in both strains. The cross-over point occurred at 32 and $52 \mathrm{~h}$

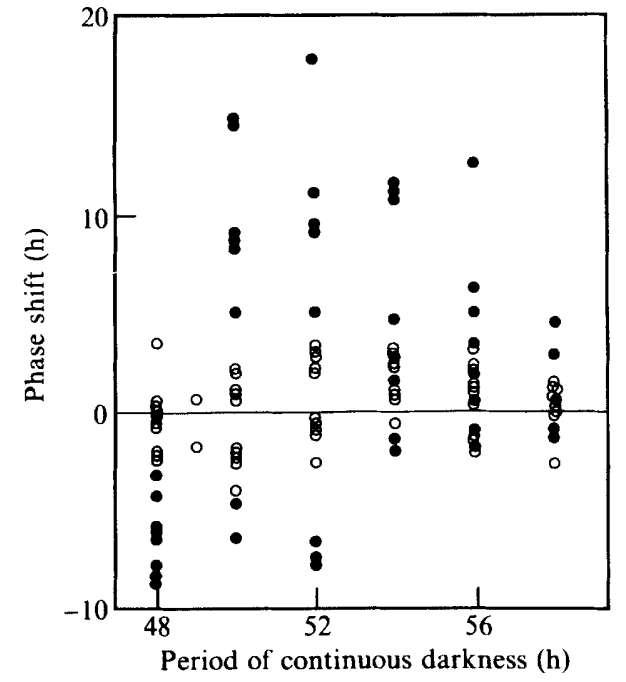

Fig. 7. Comparison of phase response curves for light among progeny of a cross between ca-23-2 and ca-23-2+. Eighteen strains were picked from the progeny of a cross between ca-23-2+ $(O)$ and ca-23-2 $(O)$. The procedure was the same as that described in the legend to Fig. 6.

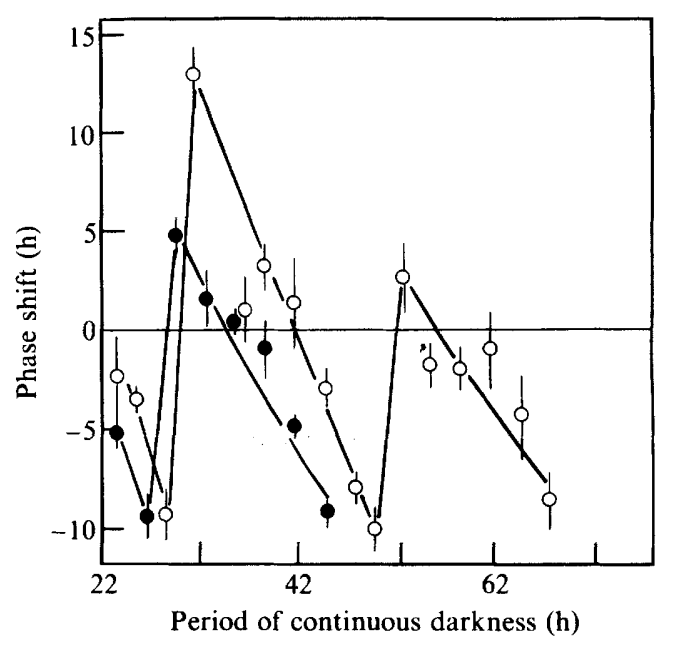

Fig. 8. Phase response curves for a $3 \mathrm{~h}$ exposure to high temperature $\left(35^{\circ} \mathrm{C}\right)$. Experimental schedules were almost the same as those described in the legend to Fig. 7 except that mycelial discs of ca-23-2+ (O) and ca-23-2 (O) were incubated at high temperature $\left(35^{\circ} \mathrm{C}\right)$ for $3 \mathrm{~h}$ and transferred to the race tubes immediately after heat treatment.

after the light-dark transition, although the extent of the maximum phase advance was different for the two strains.

\section{Discussion}

We previously described mutants that cannot grow in medium that contains a normal concentration of calcium ions (close to $1 \mathrm{mM}$ ) and concluded that the relevant 
mutation resulted in a lowering of the rate of uptake of calcium ions into vacuoles (Cornelius \& Nakashima, 1987). Strain ca-23-2 showed a completely different dependence on the external concentration of calcium ions from these earlier mutant strains. As the rate of uptake of calcium ions by ca-23-2 was same as that by the wild-type, inhibition of growth in medium containing a low concentration of calcium ions cannot be explained by a reduced rate of uptake of calcium ions.

Several mutants that showed inhibition of growth in medium with low concentrations of calcium ions have been reported in Saccharomyces. However, three mutants, cls4 (Ohya et al., 1986), call (Ohya et al., 1991) and yptI (Schmidt et al., 1988), gave completely different growth curves for growth in medium containing low concentrations of calcium ions from that of ca-23-2. Another mutant strain, pmrl, was reported to be hypersensitive to trifluoperazine and to have an extracellular invertase that has an altered molecular mass because of a change in the extent of glycosylation of this enzyme (Rudolph et al., 1989). However, ca-23-2 showed no altered sensitivity to trifluoperazine nor any change in the molecular mass of its invertase (data not shown).

Another important phenotypic difference between ca23-2 and the wild-type strain was that ca-23-2 did not show significant phase shifting by light. The irradiation that was used in these experiments was very strong compared to that necessary for a maximum shift in phase in the wild type: 10 lux of white light for $2 \mathrm{~min}$ (Nakashima, 1982). Although the culture conditions for determination of carotenoid synthesis and of lightinduced phase shifting were not the same, mycelial carotenogenesis was induced by light in both ca-23-2 and ca-23-2+, which have the same sensitivity to light. Furthermore, the phase response curve for high temperature of both strains has the same crossover point. This result indicates that the circadian clock for the conidiation rhythm of ca-23-2 runs normally. Therefore, the transduction process that connects the system for perception of light to the circadian clock must be disturbed by the mutation in ca-23-2. This result further suggests that the same reaction related to calcium metabolism is a component of this transduction process.

One of the most important characteristics of the circadian clock is that its progress is affected by changes in light or temperature. Sensitivity of the circadian clock to these stimuli changes diurnally; at some phases these stimuli exert quite dramatic effects. In particular, the diurnal change in sensitivity to light has been suggested to be a faithful reflection of the progress of the circadian clock (Pittendrigh, 1967). Therefore, analysis of the process that connects the system of light perception to the circadian clock is essential for an understanding of the molecular mechanism of the circadian clock and its regulation by light. Further analysis of the ca-23-2 strain should provide important information about this transduction process.

This work was supported in part by a grant for scientific research from the Ministry of Education, Science, and Culture of Japan and by a Naito Foundation Natural Science Scholarship.

\section{References}

Bradford, M. M. (1976). A rapid and sensitive method for the quantitation of microgram quantities of protein utilizing the principle of protein-dye binding. Analytical Biochemistry 72, 248254.

Cornelius, G. \& NaKashima, H. (1987). Vacuoles play a decisive role in calcium homeostasis in Neurospora crassa. Journal of General Microbiology 133, 2341-2347.

DAvis, R. H. \& DE SERres, F. J. (1970). Genetic and microbiological research techniques for Neurospora crassa. Methods in Enzymology 17A, 79-143.

Dharmanada, S. \& Feldman, J. F. (1979). Spatial distribution of circadian clock phase in aging cultures of Neurospora crassa. Plant Physiology 63, 1049-1054.

Eskin, A. \& CoRrent, G. (1977). Effects of divalent cations and metabolic poisons on circadian rhythm from Aplysia eye. Journal of Comparative Physiology A 117, 1-21.

Goto, K., Laval-Martin, D. L. \& Edmunds, L. N., JR (1985). Biochemical modeling of an autonomously oscillatory circadian clock in Euglena. Science 228, 1284-1288.

HARDING, R. W. (1974). The effect of temperature on photoinduced carotenoid biosynthesis in Neurospora crassa. Plant Physiology 54, 142-147.

Horowitz, N. H. (1947). Methionine synthesis in Neurospora. The isolation of crystathionine. Journal of Biological Chemistry 171, 255264.

NaKashima, H. (1981). A liquid culture method for biochemical analysis of the circadian clock of Neurospora crassa. Plant and Cell Physiology 22, 231-238.

NAKASHIMA, H. (1982). Effects of membrane ATPase inhibitors on light-induced phase shifting of the circadian clock in Neurospora crassa. Plant Physiology 69, 619-623.

NAKASHIMA, H. (1984). Calcium inhibits phase shifting by the calcium ionophore A23187 of the circadian conidiation rhythm of Neurospora crassa. Plant Physiology 74, 268-271.

Nakashima, H. (1986). Phase shifting of the circadian conidiation rhythm in Neurospora crassa by calmodulin antagonists. Journal of Biological Rhythms 1, 163-169.

OHYa, Y., MiYamoto, Y., OHSUMI, Y. \& ANRakU, Y. (1986). Calciumsensitive $c l s 4$ mutant of $S$. cerevisiae with a defect in bud formation. Journal of Bacteriology 165, 28-33.

Ohya, Y., Goeble, M., Goodman, L. E., Petersen-Bjorn, S., Friesen, J. D., TamanoI, F. \& ANRaxu, Y. (1991). Yeast $C A L I$ is a structural and functional homologue to the DPRI(RAM) gene involved in ras processing. Journal of Biological Chemistry 266, 12356-12360.

Pittendrigh, C. S. (1967). Circadian systems. I. The driving oscillation and its assay in Drosophila pseudoobscura. Proceedings of the National Academy of Sciences of the United States of America 58, 1762-1767.

Rudolph, H. K., ANtebi, A., Fink, G. R., Buckley, C. M., DoRman, T. E., Levitre, J., Davidow, L. S., MaO, J. \& MoIR, D. T. (1989). The yeast secretory pathway is perturbed by mutations in PMRI, a member of a $\mathrm{Ca}^{2+}$ ATPase family. Cell 58, 133-145.

SchmidT, H. D., Puzicha, M. \& Gallwitz, D. (1988). Study of a temperature-sensitive mutant of the ras-related YPTI gene product in yeast suggests a role in the regulation of intracellular calcium. Cell 53, 638-647. 
Slayman, C. L. \& Slayman, C. W. (1979). Whole cells for the study of transport linked to membrane potential: Neurospora crassa. Methods in Enzymology 55, 656-666.

TAMPONNET, C. \& EDMUNDS, L. N., JR (1991). Entrainment and phaseshifting of the circadian rhythm of cell division by calcium in synchronous cultures of the wild-type $\mathrm{Z}$ strain and of the $\mathrm{ZC}$ achlorophyllous mutant of Euglena gracilis. Plant Physiology 93, 425431.

VoGEL, H. J. (1956). A convenient growth medium for Neurospora (medium N). Microbial Genetics Bulletin 13, 42-43. 